\title{
Análise espacial do risco de leptospirose canina na Vila Pantanal, Curitiba, Paraná ${ }^{1}$
}

\author{
Daniele Bier ${ }^{2 *}$, Silvia E. Shimakura ${ }^{3}$, Vivien M. Morikawa², Leila S. Ullmann ${ }^{4}$, \\ Mariana Kikuti ${ }^{4}$, Hélio Langoni ${ }^{4}$, Alexander W. Biondo ${ }^{2}$ e Marcelo B. Molento ${ }^{2}$
}

\begin{abstract}
Bier D., Shimakura S.E., Morikawa V.M., Ullmann L.S., Kikuti M., Langoni L., Biondo A.W. \& Molento M.B. 2013. [Spatial analysis of the risk of canine leptospirosis in the Vila Pantanal, Curitiba, Paraná, Brazil.] Análise espacial do risco de leptospirose canina na Vila Pantanal, Curitiba, Paraná. Pesquisa Veterinária Brasileira 33(1):74-79. Departamento de Medicina Veterinária, Setor de Ciências Agrárias, Universidade Federal do Paraná, Rua dos Funcionários 1540, Curitiba, PR 80035-050, Brazil. E-mail: danielebier@gmail.com

Leptospirosis is a serious zoonotic disease associated to low income areas of urban settings. Although rodents are considered the main reservoir for urban leptospirosis, dogs may also develop the disease and become asymptomatic carriers. The objective of this study was to apply a statistical method based on the spatial point processes theory for canine leptospirosis to identify how seroreagents dogs are spatially distributed and their risk determinants in a village of Curitiba city. The model analysis allowed the identification of over-risk regions, where seropositivity risk for canine leptospirosis was significantly higher, revealing that not just one, but the combination of animal, owner and environment factors influenced the disease risk within areas with greater spatial effect. Analysis of results clearly identified the highest risk areas in the Pantanal Village, allowing the establishment of more specific preventive actions and focused on risk areas as priority for public health surveillance.
\end{abstract}

INDEX TERMS: Dogs, leptospirosis, spatial analysis, risk areas.

RESUMO.- A leptospirose é uma grave zoonose associada às áreas de baixa renda dos centros urbanos. Embora roedores urbanos sejam considerados como principal reservatório para a leptospirose, o cão também pode desenvolver a doença e se tornar carreador assintomático. 0 objetivo do presente trabalho foi utilizar a metodologia estatística baseada na teoria de processos pontuais espaciais, buscando identificar a forma como se distribuem os cães sororreagentes para a leptospirose e seus determinantes de risco em uma vila na cidade de Curitiba. A análise do modelo possibilitou identificar as regiões de sobre-risco,

\footnotetext{
${ }^{1}$ Recebido em 9 de julho de 2012.

Aceito para publicação em 13 de outubro de 2012.

${ }^{2}$ Departamento de Medicina Veterinária, Setor de Ciências Agrárias, Universidade Federal do Paraná (UFPR), Rua dos Funcionários 1540, Curitiba, PR 80035-050, Brasil.*Autor para correspondência: danielebier@gmail.com

${ }^{3}$ Departamento de Estatística, Setor de Ciências Exatas, UFPR, Cx. Postal 19081, Curitiba, PR 81531-990.

${ }^{4}$ Departamento de Higiene Veterinária e Saúde Pública, Faculdade de Medicina Veterinária e Zootecnia, Universidade Estadual Paulista (Unesp), Rubião Júnior s/n, Botucatu, SP 18618-970, Brasil.
}

onde o risco de soropositividade canina à leptospirose é significativamente maior. A relação significativa do efeito espacial no desenvolvimento da doença, além das variáveis estudadas, revela que não apenas um, mas a ação conjunta dos fatores relacionados ao animal, ao proprietário e ao ambiente influencia o risco maior da doença nos locais de maior efeito espacial. 0 resultado da análise indica claramente os territórios em maior risco na região da Vila Pantanal, possibilitando o planejamento de ações mais específicas e dirigidas a essas áreas em um contexto de vigilância da saúde.

TERMOS DE INDEXAÇÃO: Cães, leptospirose, análise espacial, áreas de risco.

\section{INTRODUÇ̃̃o}

A leptospirose é uma zoonose reconhecida como um importante problema de saúde pública mundial. A doença se estendeu além das áreas rurais e tornou-se uma das causas de epidemias em comunidades urbanas dos países em desenvolvimento (Pappas et al. 2008). Nesses países, como 
no caso do Brasil, a sua ocorrência está relacionada ao acelerado e desordenado processo de expansão urbana, onde grande parte da população passou a habitar áreas da periferia, sem o mínimo de condições de infraestrutura e saneamento, facilitando a manutenção de agentes patogênicos nocivos à saúde animal e humana.

No cão, a leptospirose constitui um grave problema de saúde pública, pois os animais que desenvolvem a doença podem tornar-se carreadores assintomáticos, assumindo então a condição de reservatório (Batista et al. 2004). Do ponto de vista epidemiológico, os cães desempenham um papel fundamental no ciclo da doença, pois se comportam como sentinelas, podendo alertar quanto à introdução de um novo sorovar de importância zoonótica, como também atuar como indicadores de contaminação ambiental (Blazius et al. 2005). A infecção humana, na maioria das vezes, está associada ao contato com água, alimentos ou solo contaminados pela urina de animais portadores da Leptospira sp.. Em situações de enchentes e inundações, a urina dos ratos, presente em esgotos e bueiros, mistura-se à enxurrada e à lama das enchentes (Bharti et al. 2003). Para os cães, os principais fatores de risco observados em estudos anteriores foram o hábito de caçar roedores, presença de áreas alagadiças próximas às residências e o acesso à rua (Querino et al. 2003).

0 estudo de aglomerados de doenças e identificação das áreas de maior risco auxilia nas tomadas de iniciativas eficazes para o controle de doenças (Bailey 2001). 0 uso dos sistemas de informações geográficas (SIG) aliado a técnicas de análise espacial permite, além do mapeamento da doença, a identificação e a avaliação de fatores de risco à saúde podendo revelar estruturas sociais, econômicas e ambientais (Barcellos e Bastos 1996). Essa abordagem oferece informações que podem favorecer possíveis planos de ação nas áreas dos estudos. Sendo assim, o desenvolvimento de estudos que utilizem ferramentas de análise espacial relacionados à leptospirose, podem favorecer a descrição de padrões de contaminação e transmissão, definir os principais fatores de risco e auxiliar a implantação de medidas e planos de ação contra essa doença.

0 objetivo do presente trabalho foi utilizar a metodologia estatística baseada na teoria de processos pontuais espaciais (Kelsall \& Diggle 1998), buscando identificar a forma como se distribuem os cães sororreagentes para a leptospirose e seus determinantes de risco em uma vila na cidade de Curitiba.

\section{MATERIAL E MÉTODOS}

A área do estudo, Vila Pantanal, está localizada no bairro Alto Boqueirão, na região sudeste de Curitiba. Esse bairro é formado por duas áreas, sendo a primeira a noroeste da linha da rede ferroviária, e a segunda área ao sudeste da linha férrea, adjacente ao Parque Estadual do Iguaçu, na qual está localizada a Vila Pantanal, objeto do presente estudo (Fig.1).

A ocupação da Vila Pantanal ocorreu no final da década de 80, na Área de Preservação Ambiental (APA) do Iguaçu, às margens do canal do Rio Iguaçu. Em 2008, habitavam no local 765 famílias (2.322 pessoas); de acordo com os dados processados em 2008 pelo Sistema de Informação da Atenção Básica, SIAB, estavam cadastradas 569 famílias $(75,9 \%)$ com 845 cães e 128 gatos. A área apresenta condições precárias de saneamento, ocorrência de constantes alagamentos e grande acúmulo de lixo. Curitiba tem um clima temperado marítimo ou clima subtropical de altitude $(C f b)$ de acordo com a classificação climática de Köppen (Peel et al. 2007), com temperatura média anual de $16^{\circ} \mathrm{C}$, caracterizado por apresentar temperaturas mais elevadas nos meses de dezembro, janeiro e fevereiro, quando também é maior a ocorrência de chuvas (IBGE 2010).

A área geográfica da Vila Pantanal foi dividida em quadras e a coleta de sangue dos cães foi realizada casa-a-casa. Participaram do estudo cães domiciliados com idade mínima de quatro meses e foram realizadas coletas durante o período de um ano (outubro de 2009 a outubro de 2010).

O sangue foi obtido por punção venosa jugular, cefálica ou safena, com agulha $30 \times 0,8 \mathrm{~mm}$ e seringa descartável de $10 \mathrm{~mL}$. Foi acondicionado em tubos de $6 \mathrm{~mL}$ para soro com gel separador, centrifugado a 3.500rpm por cinco minutos para obtenção do soro, seguido de pipetagem e acondicionado em microtubo de plástico de $2 \mathrm{~mL}$, seguido de congelamento a $-20^{\circ} \mathrm{C}$.

As amostras foram processadas e enviadas ao Núcleo de Pesquisas em Zoonoses (NUPEZO) da Universidade Estadual Paulista (Unesp) no Campus de Botucatu, para realização do teste de Soroaglutinação Microscópica (SAM), teste padrão ouro, recomendado pela Organização Mundial da Saúde. Foram utilizados os sorovares Australis, Bratislava, Autumnalis, Canicola, Cynopteri, Djasiman, Grippotyphosa, Copenhageni, Icterohaemorrhagiae, Pomona, Pyrogenes e Hardjo, totalizando doze para cada amostra

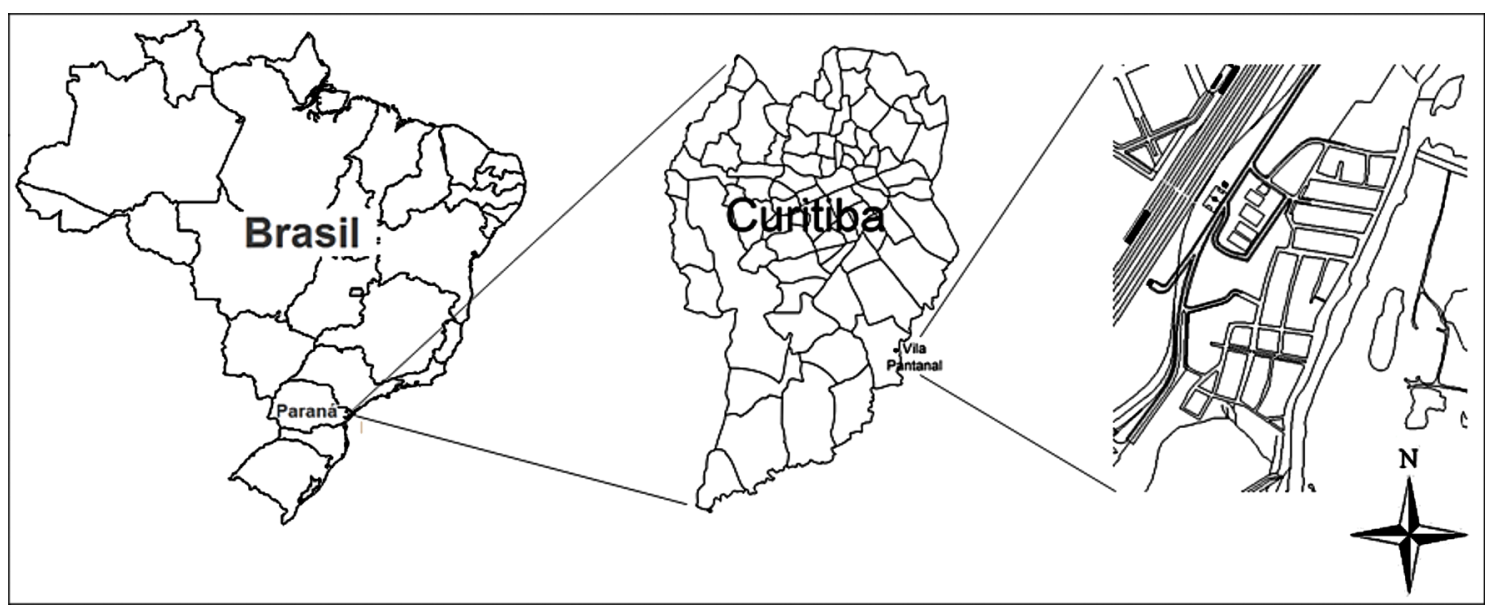

Fig.1. Localização da Vila Pantanal no Bairro Alto Boqueirão, Curitiba, Paraná, Brasil. 
testada. Foram considerados positivos títulos $\geq 100$, e a ocorrência de reação cruzada e/ou co-infecção

Foi realizada uma entrevista com os proprietários, por meio de um questionário epidemiológico, para obtenção e identificação dos possíveis fatores de risco associados à condição de animais sororreagentes para leptospirose.

As coordenadas geográficas correspondentes a cada casa em que residiam os animais do estudo foram coletadas usando um sistema de posicionamento global (Global Positioning System GPS) Geoexplorer da marca Trimble ${ }^{\circledR}$, gerando mapas geoestatísticos através do pacote geoR (Ribeiro Jr \& Diggle 2001) e o pacote mgcv (Wood 2006, Wood 2008) a fim de verificar a variabilidade espacial existente nas áreas, ambos do programa computacional R (R development core team, 2010). 0 mapa com a distribuição espacial dos cães reagentes e não reagentes à leptospirose foi gerado usando o software TerraView 4.1.0.

A estrutura de modelagem adotada nesse trabalho baseia-se em um processo pontual espacial (Diggle, 2003), pelo qual se pode definir e estimar uma medida de risco que varia continuamente na região de interesse. Para a definição da medida de risco assume-se um desenho caso-controle espacial. No presente estudo, os controles são os cães negativos para a pesquisa sorológica da leptospirose. A estimação da medida de risco avalia simultaneamente a variação na distribuição espacial de casos (cães soropositivos) quando comparados à distribuição espacial de controles (cães soronegativos). 0 presente trabalho utiliza a abordagem de Modelos Aditivos Generalizados (Generalized additive model GAM) proposta por Kelsall \& Diggle (1998), que permite a estimação espacial do risco controlando por fatores individuais de risco. As 18 variáveis utilizadas estão descritas no Quadro 1. Quatro delas são características relacionadas com os moradores da casa; cinco são relacionadas com os cães; e nove são relacionadas com o ambiente. Com exceção das variáveis sexo, rede de esgoto ou fossa, quantas pessoas e quantos cães, que podiam apresentam mais de um valor, as demais variáveis foram categorizadas com SIM e NÃO.

Além do mapeamento do risco, buscou avaliar se a superfície estimada variou significativamente na região, ou seja, reconhecendo o papel de dado fator como preditor importante da leptospirose canina e controlando por este, deseja-se identificar áreas nas quais o risco é significativamente elevado, buscando orientar a intervenção. 0 teste global do risco e a identificação de áreas de

\section{Quadro 1. Descrição das variáveis utilizadas}

\section{Variáveis relacionadas com os moradores da casa}

ConheceLepto Se algum morador da casa conhecia a leptospirose AlguemLepto Se algum morador da casa foi diagnosticado com leptospirose Qtaspessoas Quantas pessoas moram na casa Qtoscães Quantos cães residem na casa

Variáveis relacionadas com os cães

\begin{tabular}{|c|c|}
\hline & \\
\hline ContCaes & Se existe contato do cão com outros cães \\
\hline Raca & Qual a raça do cão \\
\hline Sexo & Qual o sexo do cão \\
\hline AcessoRua & Se o cão tem acesso à rua \\
\hline PoteReco & Se o pote de ração e água são recolhidos à noite \\
\hline Jariáveis rela & onadas com o ambiente \\
\hline Ratos & Se há presença de ratos na casa ou próximo ao domicílio \\
\hline Entulho & Se há entulho dentro do domicílio \\
\hline Recicláveis & Se há recicláveis dentro da casa ou terreno \\
\hline Esgoto & Se há esgoto a céu aberto próximo ou dentro do domicílio \\
\hline Enchente & Se já houve enchente na casa \\
\hline Terra & Se a casa possui terra no terreno \\
\hline Vegetação & Se a casa possui algum tipo de vegetação no terreno \\
\hline Cimento & Se a casa é cimentada \\
\hline
\end{tabular}

EsgotoOuFossa Se o esgoto da casa é ligado à rede ou se há fossa na casa baixo e alto risco foram realizados utilizando o método de simulação Monte Carlo, proposto por Kelsall \& Diggle (1998), adicionando os contornos de 0.05 e 0.95 da superfície de p-valores ao mapa.

0 presente estudo foi submetido e aprovado pela Comissão de Ética no Uso de Animais do Setor de Ciências Agrárias, Universidade Federal do Paraná (Protocolo 007/2009).

\section{RESULTADOS}

Nos testes de sorodiagnóstico para leptospirose realizados, das 378 amostras, $105(27,8 \%)$ foram positivas para pelo menos um dos 12 sorovares testados, considerando-se os títulos acima de 100 e a ocorrência de reação cruzada e co-infecção, ou seja, que um mesmo animal pudesse estar infectado com mais de um sorovar. Todos os cães foram georreferenciados na posição dos seus domicílios. A Figura 2 apresenta a distribuição espacial dos cães amostrados, a localização geográfica dos cães negativos à SAM é representada por pontos verdes e os positivos por pontos vermelhos.

O modelo final ajustado, por critério de informação de Akaike (Hastie \& Tibshirani 1990), assume um efeito linear do total de oito variáveis no logaritmo do risco. As estimativas dos parâmetros do modelo controlando-se o fator espacial são apresentadas no Quadro 2. Dentre as variáveis, apenas a característica relacionada ao animal (se não possuía raça) mostrou-se significativamente associada à soropositividade do cão à leptospira. As demais variáveis não apresentaram associação estatisticamente significativa entre a população estudada.

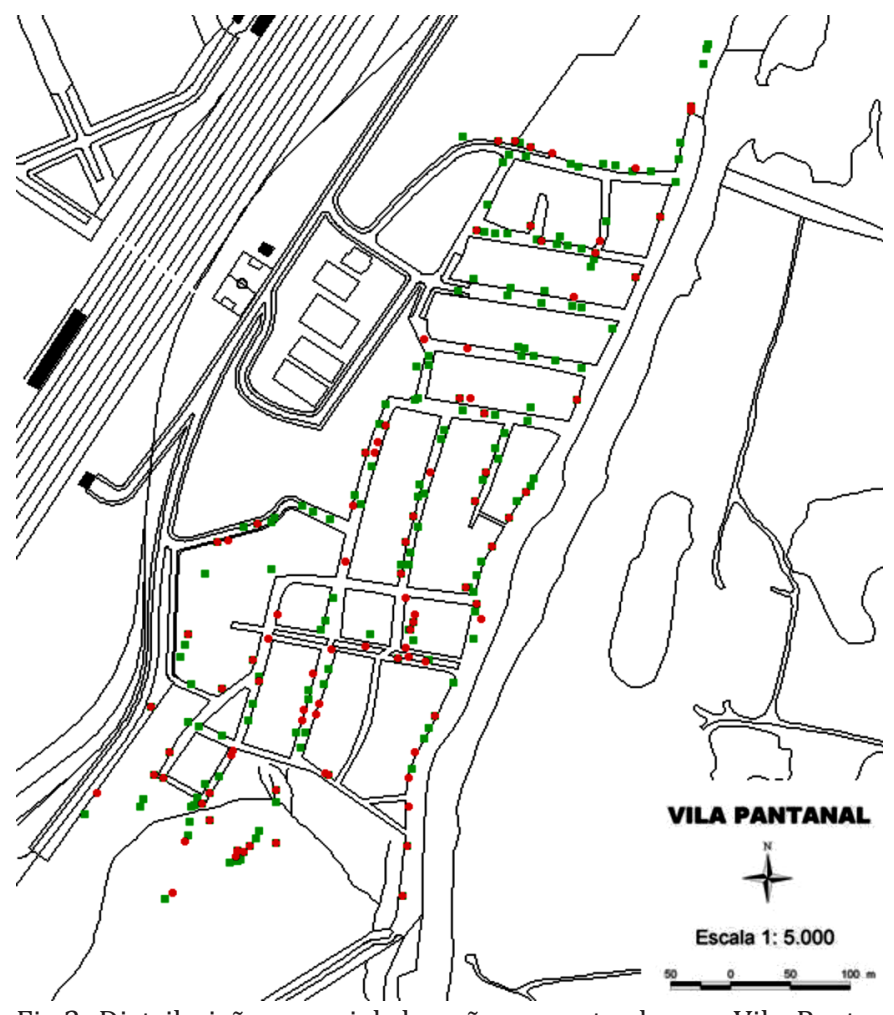

Fig.2. Distribuição espacial dos cães amostrados na Vila Pantanal, Curitiba, PR, no período de outubro de 2009 a outubro de 2010. A localização geográfica dos cães negativos a soroaglutinação microscópica é representada por pontos verdes e os positivos por pontos vermelhos. 
Quadro 2. Estimativas dos efeitos de variáveis

\begin{tabular}{lccc}
\hline \multicolumn{1}{c}{ Fatores } & Estimativa & Erro Padrão & P-valor \\
\hline Ratos (Sim) & -0.2121 & 0.2513 & 0.3987 \\
Sem raça definida (Sim) & 0.7064 & 0.3511 & 0.0442 \\
Esgoto (Sim) & -0.1408 & 0.2681 & 0.5994 \\
Pote recolhido (Sim) & 0.1919 & 0.2512 & 0.4449 \\
Rede de esgoto ou fossa (Nenhum) & -0.3940 & 0.3725 & 0.2901 \\
Rede de esgoto ou fossa (RedeEsgoto) & -0.1727 & 0.3178 & 0.5868 \\
Enchente (Sim) & 0.2073 & 0.3294 & 0.5291 \\
Cimento (Sim) & -0.3931 & 0.2530 & 0.1202 \\
Recicláveis (Sim) & 0.2679 & 0.4723 & 0.5706
\end{tabular}

0 efeito espacial controlando-se por fatores individuais para a ocorrência de soropositividade à leptospirose canina sobre a Vila Pantanal, em Curitiba, está apresentado na Figura 3 e indica variação espacial significativa no risco com p-valor de 0,039. Como este efeito não tem uma escala de valores de interpretação direta, foi calculado um risco relativo (Fig.4) sob a hipótese de homogeneidade espacial.

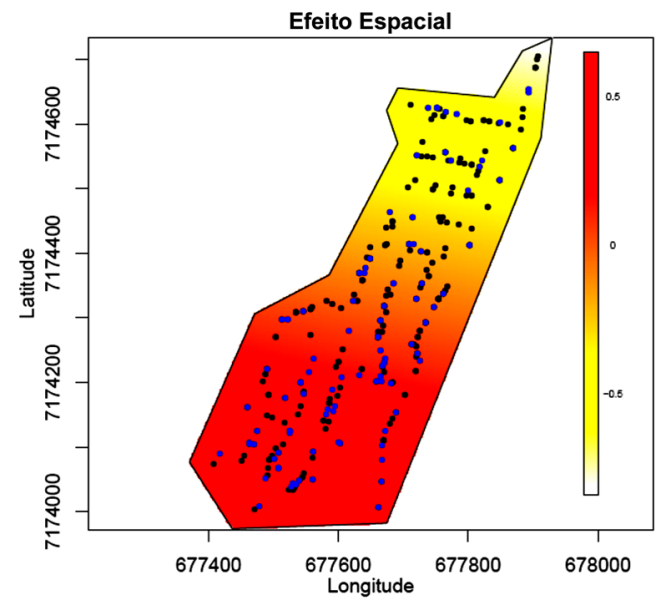

Fig.3. Mapa do efeito espacial e localização dos animais soropositivos (azul) e soronegativos (preto) à leptospirose.

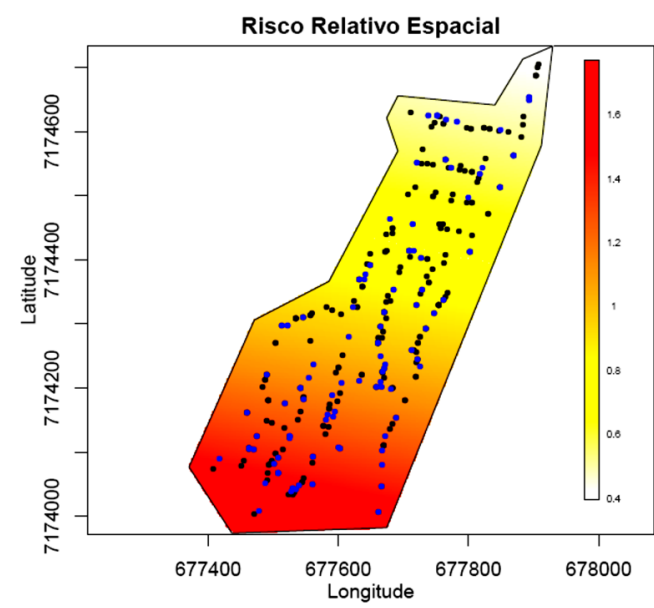

Fig.4. Risco relativo para a leptospirose canina e localização dos animais soropositivos (azul) e soronegativos (preto). 0 valor da escala representa o número de animais positivos acima da média. Por exemplo, nos locais com a cor representada pelo valo de referência 1.6, demonstra que essas áreas têm quase o dobro (1,6 vezes mais) de animais soropositivos do que a média geral da área.

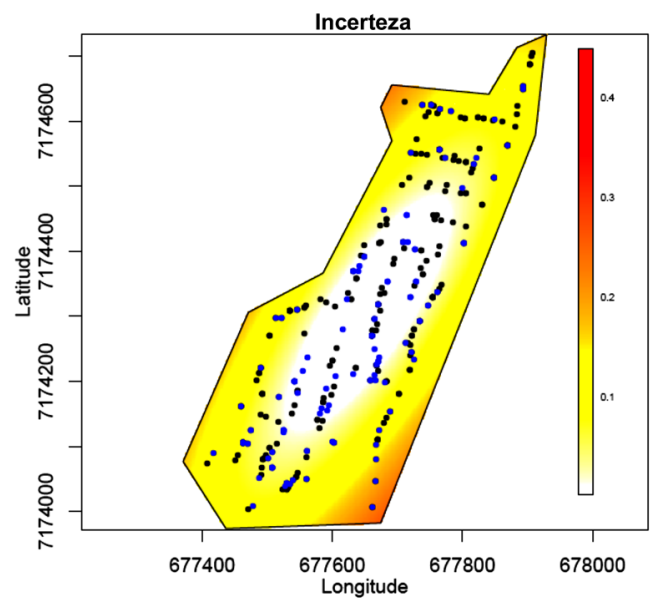

Fig.5. Incerteza associada ao risco relativo espacial e localização dos animais soropositivos (azul) e soronegativos (preto) à leptospirose. 0 menor erro padrão $(=0)$ é apresentando no mapa em cor branca, aumentando o erro de acordo com a intensidade da cor.

Para isto considera-se que a área é homogênea e obtém-se uma média de todas as predições, depois se divide cada uma das predições pela média geral. Com isto é possível avaliar o risco relativo em relação à média geral em que observações próximas da média apresentarão valores próximos da unidade. A Figura 5 mapeia a incerteza associada a cada predição medida pelo erro padrão de predição em cada ponto. Com o mapa de risco relativo espacial é possível identificar áreas com risco relativo acima de um valor de referência, por exemplo, 1.6, mostrando que essas áreas têm quase o dobro de animais soropositivos do que a média geral da área. Isto permite delimitar áreas de alta infecção pela Leptospira sp., e que devem receber maior atenção pelas agências de saúde locais. No mapa de erros padrão fica evidente que a incerteza aumenta em localizações onde existem poucas observações. Por exemplo, pequenas bordas do mapa de incerteza apresentam um erro padrão de aproximadamente 0.25 , enquanto que onde foram feitas as observações o erro padrão varia entre 0 e 0.1 .

\section{DISCUSSÃO}

A soroprevalência apresentou um padrão de distribuição espacial que envolveu toda a área da Vila Pantanal. Pode-se deduzir que, na escala abordada, houve correlação espacial com características epidemiológicas, uma vez que toda a Vila apresenta situação de risco para contaminação por Leptospira spp., assim como o observado por Magalhães et al. (2006) que verificaram concentração de cães sororreagentes em áreas de saneamento básico deficiente.

Para a análise espacial da leptospirose em nível municipal devem ser levados em consideração três fatores principais: as condições sociais dessa população, o risco de ocorrência de inundação e a concentração populacional (Paula, 2005). Analisando-se conjuntamente esses três aspectos, torna-se evidente o motivo pelo qual a Vila Pantanal apresentou grande distribuição de animais reagentes para leptospirose. A alta densidade demográfica e a crítica condição de pobreza, aliadas ao fato de que diversas regiões da 
Vila estão sujeitas às inundações, deixam o local com condições favoráveis à disseminação da doença. Para Sabroza et al. (1992), a maneira como os espaços são ocupados por populações de diferentes estratos socioeconômicos pode tornar tais espaços vulneráveis e criar condições favorecedoras para produção e reprodução de doenças.

Através do modelo empregado foi possível obter estimativas do risco continuamente no espaço, controlando por fatores individuais de risco e utilizando as localizações pontuais de casos e controles. Para a soropositividade canina à leptospirose na Vila Pantanal, das diversas variáveis analisadas, no modelo melhor ajustado, apenas a característica relacionada ao animal, se não possuía raça mostrou-se significativamente associada. Entretanto, a raça do animal tem pouca influência quando se trata da ocorrência de leptospirose canina. Diversos trabalhos também demonstram uma maior proporção de cães sem raça definida reagentes, porém sem significância estatística (Caldas et al. 1977, Modolo et al. 2000, Batista et al. 2005, Silva et al. 2006). No presente trabalho, essa característica encontrada pode estar relacionada ao número muito grande de animais sem raça definida $(\mathrm{n}=312)$, quando comparado a os de raça $(n=66)$ analisados.

Quando se realiza o mapeamento do risco, observa-se a relação significativa do efeito espacial no desenvolvimento da doença, além das variáveis estudadas, reconhecendo que existe o papel de algum fator como preditor importante da leptospirose canina, o qual não foi um dos analisados pelo presente estudo. Ou seja, pode haver outras variáveis não levadas em consideração neste trabalho que devem ser importantes para explicar a resposta. Tais variáveis teriam padrão espacial de distribuição na área e, na ausência de medidas, têm seus efeitos capturados pelo efeito espacial. Certamente, não apenas um, mas a ação conjunta desses fatores influencia o risco maior da doença nos locais de maior efeito espacial e, consequentemente, maior risco relativo espacial.

A análise do modelo possibilitou identificar as regiões de sobre-risco, onde a soropositividade canina à leptospirose é significativamente maior. Essas superfícies de risco diferenciado relacionam-se às condições socioeconômicas e ambientais das áreas, independente da ocorrência individual das variáveis. Ou seja, os animais e as pessoas moradoras nessas regiões estão expostos a situações de risco coletivo que não aquelas indicadas pelas variáveis analisadas. Através desse mapeamento do risco, foi possível identificar que as áreas nas quais o risco é significativamente elevado, coincidiram com as áreas de pior condição socioeconômica e ambiental, que revelou a presença de esgotos a céu aberto dentro e próximo dos domicílios, lixos e entulhos à revelia nos quintais das casas e cães semi-domiciliados com acesso à rua.

Na falta de uma política habitacional capaz de atender às pressões urbanas das grandes cidades, multiplicaram-se as ocupações irregulares de terras e assentamentos precários ou favelas (Pimentel et al. 2000). Os moradores dessas regiões acabam despejando seu lixo onde lhes for conveniente, ficando de fácil acesso para os cães e roedores, o que cria condições favoráveis para a disseminação da leptospirose. Diversos trabalhos, que também analisaram o contexto ambiental de casos de leptospirose, verificaram que as maiores taxas de incidência ocorreram nas regiões ao redor das zonas de acumulação de lixo e de deficiência de infraestrutura básica, apontando para a combinação de fatores socioambientais, ainda que a relação não seja direta (Barcellos e Sabroza 2000; Figueiredo et al. 2001, Romero et al. 2003, Dias et al. 2007, Soares et al. 2010).

0 resultado da análise indica, claramente, os territórios em maior risco na região da Vila Pantanal, possibilitando o planejamento de ações mais específicas.

\section{CONCLUSÕES}

Através da utilização dos resultados sorológicos de um grande número de cães dentro de uma comunidade urbana carente do Município de Curitiba, este trabalho mostrou ser possível obter estimativas do risco continuamente no espaço, controlado por fatores individuais de risco e utilizando as localizações pontuais dos casos e controles.

As técnicas de análise espacial utilizadas ajudaram a compreender melhor a distribuição dos animais sororreagentes à leptospirose e identificar os territórios em maior risco na região da Vila Pantanal, possibilitando o planejamento de ações mais específicas e dirigidas a essas áreas em um contexto de vigilância da saúde.

\section{REFERÊNCIAS}

Bailey T.C. 2001. Spatial statistical methods in health. Cad. Saúde Pública 17:1083-1098.

Barcellos C. \& Bastos F.I. 1996. Geoprocessamento, ambiente e saúde: uma união possível? Cad. Saúde Públ. 12: 389-397.

Barcellos C. \& Sabroza P. 2000. Socio-environmental determinants of the leptospirosis outbreak of 1996 in western Rio de Janeiro: A geographical appoach. Int. J. Health Res. 10:301-313.

Batista C.S.A., Alves C.J., Azevedo S.S., Vasconcellos S.A., Morais Z.M., Clementino I.J., Alves F.A.L., Lima F.S. \& Araújo Neto J.O. 2005. Soroprevalência e fatores de risco para a leptospirose em cães de Campina Grande, Paraíba. Arq. Bras. Med. Vet. Zootec. 57:179-185.

Batista C.S.A., Azevedo S.S., Alves C.B., Vasconcellos A.S., Morais Z.M., Clementino I.J, Lima F.S. \& Araújo Neto J.O. 2004. Soroprevalência de leptospirose em cães errantes da cidade de Patos, Estado da Paraíba, Brasil. Braz. J. Vet. Res. Anim. Sci. 41:131-136.

Bharti A.R., Nally J.E., Ricaldi J.N., Matthias M.A., Diaz M.M., Lovett M.A., Levett P.N., Gilman R.H., Willig M.R., Gotuzzo E. \& Vinetz J.M. 2003. Leptospirosis: A zoonotic disease of global importance. Lancet, Infect Dis. 3:757-771.

Blazius R.D., Romão P.R.T., Blazius E.M.C.G. \& Silva O.S. 2005. Ocorrência de cães errantes soropositivos para Leptospira spp. na cidade de Itapema, Santa Catarina, Brasil. Cad. Saúde Públ. 21:1952-1956.

Caldas E.M., Doria J.D. \& Martins M.A. 1977. Immunological inquiry for the epidemiology of leptospirosis in canis familiaris in Salvador, Bahia, Brazil. Int. J. Zoonoses 4:103-110.

Dias J.P., Teixiera M.G., Costa M.C.N., Mendes C.M.C.M., Guimarães P., Reis M.G., Ko A.I. \& Barreto M.L. 2007. Factors associated with Leptospira sp. infection in large urban center in northeastern Brazil. Revta Soc. Bras. Med. Trop. 40:499-504.

Diggle P.J. 2003. Statistical Analysis of Spatial Point Patterns. $2^{\text {nd }}$ ed. Arnold, London. 159 p.

Figueiredo C.M., Mourão A.C., Oliveira M.A.A., Alves W.R., Ooteman M.C., Chamone C.B. \& Koury M.C. 2001. Leptospirose humana no município de Belo Horizonte, Minas Gerais, Brasil: uma abordagem geográfica. Rev. Soc. Bras. Med. Trop. 34:331-338. 
Hastie T. \& Tibshirani R. 1990. Generalized Additive Models. Chapman and Hall, London. 335p.

IBGE 2010. Censo Demográfico: Cidades - Curitiba. Instituto Brasileiro de Geografia e Estatística. Available from <http://www.ibge.gov.br/cidadesat/topwindow.htm?1> Accessed on April 20, 2010.

Ihaka R. \& Gentleman R. 1996. R: A Language for Data Analysis and Graphics. J. Comp. Graph. Stat. 5:299-314.

Kelsall J.E. \& Diggle P.J. 1998. Spatial variation in risk of disease: A nonparametric binary regression approach. Appl. Stat. 47:559-573.

Magalhães D.F., Silva J.A., Moreira E.C., Wilke V.M.L., Haddad J.P.A. \& Meneses J.N.C. 2006. Prevalência de aglutininas anti-Leptospira interrogans em cães de Belo Horizonte, Minas Gerais, 2001 a 2002. Arq. Bras. Med. Vet. Zootec. 58:167-174.

Modolo J.R., Langoni H., Shimabukuro F.H., Mendonça A.O., Victória C. \& Padovani C.R. 2000. Inquérito soroepidemiológico para leptospirose canina, no município de Botucatu/SP. Anais 27ํㅡㄹ Congresso Brasileiro de Veterinária, Águas de Lindóia, SP, p.95. (Resumo)

Pappas G., Papadimitriou P., Siozopoulou V., Christou L. \& Akritidis N. 2008. The globalization of leptospirosis: worldwide incidence trends. Int. J. Infect. Dis. 12:351-357.

Paula E.V. 2005. Leptospirose Humana: uma análise climato-geográfica de manifestação no Brasil, Paraná e Curitiba. Anais XII Simpósio Brasileiro de Sensoriamento Remoto, Goiânia, Goiás, p.2301-2308. (Resumo)

Pimentel A.M., Régis F.J.M. \& Carneiro R.M. 2000. Leptospirose, p.481-484 In: Tonelli E. \& Freire L.M.S. (Eds), Doenças Infecciosas na Infância e na Adolescência. Editora Medsi, Rio de Janeiro.
Querino A.M.V., Delbem A.C.B., Oliveira R.C., Silva F.G., Müller E.E., Freire R.L. \& Freitas J.C. 2003. Fatores de risco associados à leptospirose em cães do município de Londrina/PR. Semina: Ciências Agrárias 24:27-34.

R Development Core Team 2010. R: A language and environment for statistical computing. R Foundation for Statistical Computing, Vienna, Austria. ISBN 3-900051-07-0, URL http://www.R-project.org.

Ribeiro Jr P.J. \& Diggle P.J. 2001. GeoR: A package for geostatistical analysis. R-NEWS. 1:15-18.

Romero E.C., Bernardo C.C.M. \& Yasuda P.H. 2003. Human leptospirosis: a twenty-nine-year serological study in São Paulo, Brazil. Revta Inst. Med. Trop., São Paulo, 45:245-248.

Sabroza P.C., Toledo L.M. \& Osanai C.H. 1992. A Organização do espaço e processos endêmicos-epidêmicos, p.57-77. In: Leal M.C., Sabroza P.C., Rodrigues R.H. \& Bus P.M., (Eds), Saúde, Ambiente e Desenvolvimento. Hucitec, São Paulo/Abrasco, Rio de Janeiro.

Silva W.B., Simões L.B., Lopes A.L.S., Padovani C.R., Langoni H. \& Modolo J. R. 2006. Avaliação de fatores de risco de cães sororreagentes à Leptospira spp. e sua distribuição espacial, em área territorial urbana. Braz. J. Vet. Res. Anim. Sci. 43:783-792.

Soares T.S., Latorre M.D.O.R., Laporta G.Z. \& Buzzar M.R. 2010. Spatial and seasonal analysis on leptospirosis in the municipality of São Paulo, Southeastern Brazil, 1998 to 2006. Revta Saúde Públ. 44:283-91.

Wood S.N. 2006. Generalized Additive Models: An introduction with R. Chapman and Hall/CRC, Boca Raton, Florida. 120p.

Wood S.N. 2008. Fast stable direct fitting and smoothness selection for generalized additive models. J. Roy. Stat. Soc. B 70:495-518. 